\title{
Automatic Inspection System for Quality Evaluation of Fresh Tuna Meat
}

\author{
A. Mateo, F. Soto, J.A. Villarejo, C. Fernandez \\ Department of Electronic Technology (DTE) \\ Technical University of Cartagena \\ Campus Muralla del Mar s/n. Cartagena, Spain, E-30202 \\ Email: antonio.mateo@upct.es, pencho.soto@upct.es, jose.villarejo@upct.es, carlos.fernandez@upct.es
}

\begin{abstract}
This paper describes an Automated Visual Inspection System SIVATUN ${ }^{\mathrm{TM}}$ for quality control of Tuna Meat which automate manual inspection operation. Depending on whether tuna are reared in cages or in the open sea and depending on the method of capture (electroslaughtering, shooting, etc.), differences are observed and changes take place in the organic nature of Blue Fin Tuna (Thunnus Thynnus) meat, affecting appearance and quality. These changes are caused by an increase and accumulation of lactate, which negatively affects the quality. This drop in quality translates into loss of value of the meat in Japanese tuna markets (Tsukiji market and Sashimi market are the main destinations of tuna captured off the southeast coast of Spain). In order to evaluate these changes, Japanese experts are employed to carry out visual inspections, which therefore constitute a subjective assessment. This paper describes the development of an automated visual inspection system that can analyze, model and detect these changes. The ultimate aim is to establish quality indicators and classifiers that will accompany tuna meat from the time of capture and so make it possible to track this product in the main tuna meat export markets. In this way we can establish a connection between variation of tuna meat quality and the feeding and slaughtering methods used, thus providing feedback to the fattening and slaughtering processes so as to improve the global quality of Blue Fin Tuna catches.
\end{abstract}

\section{INTRODUCTION}

Tuna farming is defined as rearing tuna for a short period in order to increase its commercial value by increasing fat contents. There have been several trials of bluefin aquaculture, in which tuna are kept for a few years in a cage until they reach a good commercial size. The concept of aquaculture is different from that of farming, and they must therefore be considered separately. Originally, large but lean bluefin tuna (generally post-spawning) captured by trap fishing were kept in a cage for a few months, during which they were fed with bait to increase their fat contents. Almost all these tuna were shipped to the Japanese market, particularly when prices were at their peak (i.e. towards the end of the year). In this way, the value of tuna sometimes increased more than a hundred fold in a matter of months [14].

Because of the strong Japanese demand for fresh tuna for raw consumption in the form of sushi or sashimi, control of the final quality of this product is extremely important. The factors that determine final quality in the Japanese market are: freshness, fat content, meat color and absence of Yake a Japanese term for BTS (Burn Tuna Syndrome). Yake may be the result of any of three factors: high body temperature, acid lactic production or high proteolytic activity [8].

It has been demonstrated that vigorous activity during the capture of BFT affects the meat quality [11]. To avoid activity, a new slaughtering system is being used; this entails administration of an electrical discharge by an electronic device [13], causing instantaneous death and considerably reducing the activity and stress of tuna. Otherwise, this would result in anaerobic metabolism with a rapid decrease in the $\mathrm{pH}$ value of the meat as a consequence of high acid lactic production [12]; this builds up in the tuna muscle, leading to the appearance of Yake, which affects muscle strength and can cause the meat to break up more easily in movement [11].

In the processing industries, color cards, color meters and other techniques are used for the standardization of color in terms of quality, principally for salmonids [18]. However, human assessment of color is subjective and may be affected by differences in color perception and lighting conditions [1]. This entails the possibility of errors in the subjective determination of quality as well as making it necessary for assessors to travel from the country of destination of the product (usually Japan) to inspect and assess tuna meat characteristics after slaughter.

Visual color inspection systems have been used to analyze a variety of food items such as fish meat fillets [10], shrimp [7], [3], beef and carrots [1], skin color of Goldfish [6], and ox meat quality [17]. A computerized vision system offers objective measurements under uniform conditions and can analyze a much larger surface area than the traditional colorimeter. Fresh tuna meat quality is normally determined by visual inspection of tuna samples in the processing plant by trained personnel (graders). These are usually assessors from Japan. These assessors determine the different levels of quality exhibited by tuna samples for every day's catch.

This article presents a new application of an automated visual inspection system for measuring and analyzing the color and texture of tuna meat after slaughter and prior to commercialization. These data are essential for the sale and grading of tuna meat in the demanding Japanese market, which is the principal destination of this product. $\Delta \mathrm{E}$ parameter estimate [16] and gray level co-occurrence matrix GLCM [9] 
techniques are used. Estimation of GLCM for gray-level texture discrimination, was used in this application to determine and model the color co-occurrence on tuna meat images as well as the Homogeneity Histogram segmentation technique [2] to determine color and localized fat distribution.

\section{THE DEVELOPED SYSTEM}

For analysis of tuna samples, a processing area must be defined in which the situation of elements for analysis (barcode, tail-type and back-type samples) is determined, and also an appropriate lighting system. In the SIVATUN structure (Fig.1), in addition to the inspection area, there is a visualization zone containing a touch screen for control of CMVS operations, and a hardware area containing the $\mathrm{PC}$ and other control elements. The lighting system consists of a bellshaped PVC canopy, which provides suitably diffuse illumination of the inspection area to eliminate glinting from the highly reflective surfaces that make up this area.

Image acquisition is by a CCD (charge coupled display) color camera placed in the upper part of the bell structure, with $1016 \times 768$ pixels resolution and $16 \mathrm{~mm}$ optics giving a visible area of $22 \times 17 \mathrm{~cm}$. On this surface is a PMMA (Poly Methyl Methacrylate) frame which divides the analysis area into three parts. One of these parts is designed to hold a tail-type sample, another a back-type sample, and the third a barcode generated in the processing plant with information on the tuna from which the sample was taken.

In the analysis area for back-type samples, there is an LEDbased lighting system (Fig. 2) which illuminates the sample perpendicular to the camera's line of vision. This lightning system is so configured as to ensure availability of the visual information needed to evaluate the transparency or translucence of the tuna back-sample. The background of this zone is colored black for effective segmentation of the sample holding area.

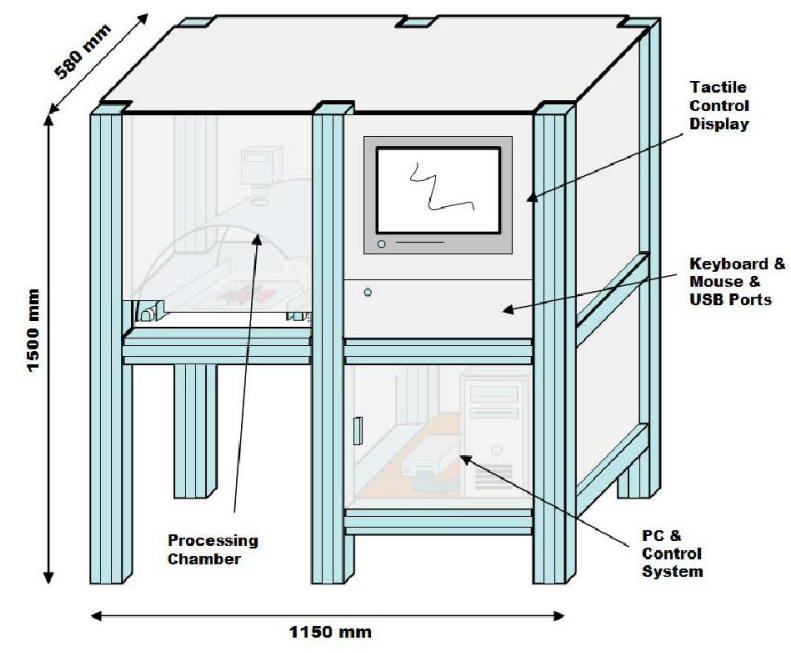

Fig 1: General configuration of SIVATUN system structure.

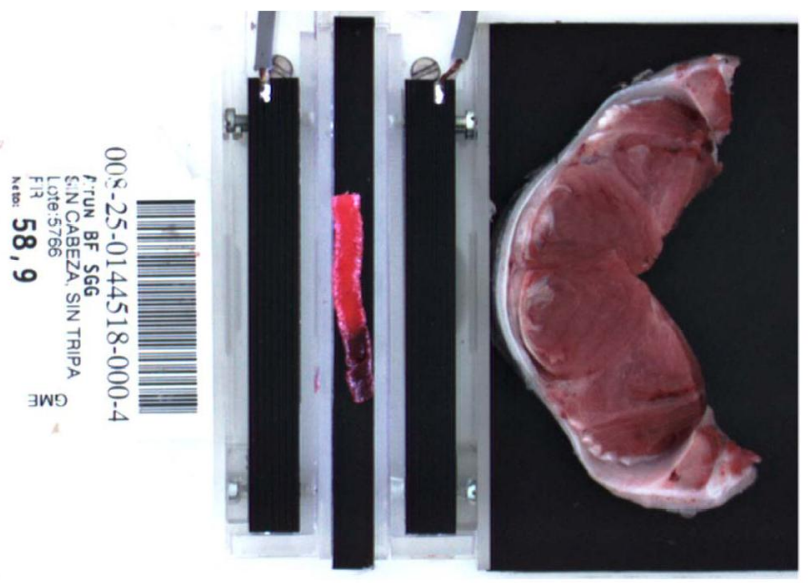

Fig 2: CCD camera-captured image of the inspection area. The target elements are, from left to right: barcode; back-type sample placed between the LED linear lighting elements that provide a beam of linear light of $90 \mathrm{~mm}$; and tailtype sample.

The translucence, which can be seen in the images in Fig. 3, is appreciable in the variation of the lightness $\left(\mathrm{L}^{*}\right)$ and chroma $\left(a^{*}, b^{*}\right)$ parameters of the system CIE $L^{*} a^{*} b^{*}$ used for the description of color. This variation is correlated with an increase or decrease of tuna quality according to the graders' assessments of samples. This feature can be observed in Fig. 4 by comparing the tendency of the slope of "Graders Quality" with the CIE L*, CIE $a^{*}$ CIE $b^{*}$ values.

\section{IMAGE ANALISIS}

\section{A. Color analysis by $\triangle E$ parameter estimation}

This technique is based on conversion of the standard RGB uniform color space representation to the CIE L*a* $\mathrm{b}^{*}$ (1976) standard. The latter is better suited to the determination of differences between colors as it presents more uniform perceptual properties than others representational spaces [16].
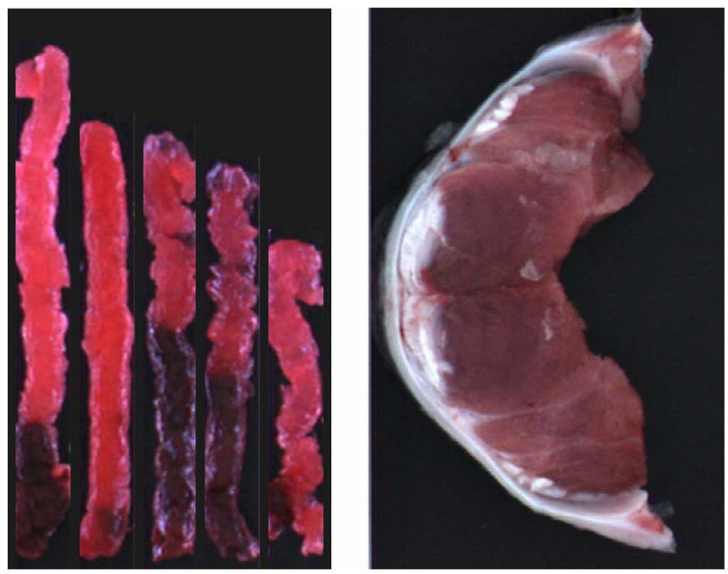

Fig. 3. Different shapes and color intensities presented by back-type samples (left). This feature indicates translucence, which is appreciable thanks to the linear LED lighting system that penetrates the samples. The shape and appearance of tail-type samples is shown on the right. 
Therefore the difference between two colors is obtained by calculating the $\triangle \mathrm{E}$ parameter using the CMC an CIEDE2000 formulation with two points of color plotted in the CIE L*a*b* uniform space, to give the parameter denominated $\Delta \mathrm{E}$. From this parameter scientists can derive the JND (Just Noticeable Difference) value and use that as the basic unit of color (one $\mathrm{JND}=\Delta \mathrm{E} / 2.3$ ). This parameter provides the minimum differentiable value, which is a function of the total distance between two colors $\Delta \mathrm{E}$.

The CIE $\mathrm{L}^{*} \mathrm{a}^{*} \mathrm{~b} *$ color value measured in the target tuna sample is compared with the database of quality grade model values previously stored in data files used by the SIVATUN application. This comparison yields a JND parameter with respect to each of these $\mathrm{L}^{*} \mathrm{a}^{*} \mathrm{~b}^{*}$ model values. Finally, quality is determined as the grade for which the JND value is smallest.

\section{B. Statistical analysis by gray level co-ocurrence matrix technique}

The GLCM texture analysis technique [9] consists in an analysis of the co-occurrence of gray levels in the pixels of an image for a specific spatial direction and pixel distance, with the aim of identifying a matrix that represents the color texture distribution model of the target by matching each RGB color space channel [4]. In this case, due to the narrow range of the color scheme and the small size of the tuna sample images, the technique was modified by introducing a bilinear interpolation operation to expand the image before the GLCM matrix was generated. In this way more information was obtained about the color texture [15]. This procedure was followed for each one of the single channels of the RGB and CIELab color spaces and for the multichannel combination of its color spaces (RG,RB,GB and La,Lb,ab) [4].

Estimation of the gray level co-occurrence matrix models the texture pattern of the image and the color texture of the model samples for each color channel, providing a set of benchmark matrixes for each quality grade defined.

If a sample is representative of a given quality grade, it is analyzed by the CMVS using $\triangle \mathrm{E}$ and GLCM procedures. The models thus produced will be assigned the quality value awarded that sample by the expert graders, and stored in a quality database. The quality of an ungraded tuna sample is determined by comparison of the GLCM matrix of the tuna sample image with the previously-established GLCM modelmatrix quality database. This comparison is set for each color channel combination (see Table I).

The similarity of the model of an ungraded sample to each of the pre-established quality models, $\mathrm{Si}$, is determined according to (1), in which $M_{i}$ is the GLCM of each of the quality grade model images and $X$ is the GLCM of the ungraded tuna sample image.

The quality grade is set to $M_{i}$, if $S_{j}>S_{i}$ with $(j \neq i)$.

$$
S_{i}=\frac{X \cdot M_{i}}{|X| \cdot\left|M_{i}\right|}
$$

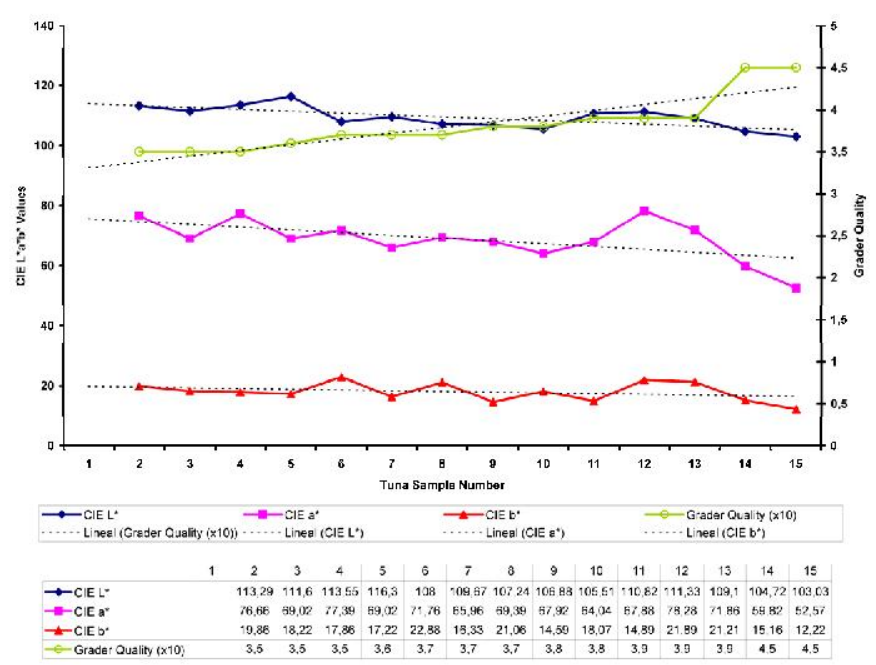

Fig. 4. Results of measurement of Lightness and Chroma parameters of the CIE L*a*b* color space in a set of 15 tuna samples. Results appear in increasing order of magnitude of the quality grading awarded by the expert graders for every tuna sample. Lightness $\left(\mathrm{L}^{*}\right)$ and Chroma $\left(\mathrm{a}^{*} \mathrm{~b}^{*}\right)$ values decrease when the quality grade increases. The table at the bottom of the figure shows the numerical values of these parameters for each tuna sample.

\section{Homogeneity Histogram color segmentation technique}

Following analysis of back-type sample, the next step is to analyze the tail-type sample to determine the localized fat content and its distribution as indicated by areas of similar color observed in the sample.

To do this, the homogeneity histogram technique [2], adapted to the special characteristics of this application, is applied to the tail-type sample images. This includes the use of thresholds based on detection of significant peaks and valleys. In this way a distribution of uniform color areas can be established with more or less segmentation depending on the level specified in the algorithm, which can readily be configured from the SIVATUN main window.

The distribution of uniform colors is plotted as a percentage of the total area of the tail-type sample. This information is shown in a specific area of the graphic interface of the SIVATUN application (Fig. 5) and included in the data file of results of analysis of the sample concerned (Table I).

In this way a segmented image is obtained with perfectly delimited areas of uniform color usually comprising 5 or 6 regions. The color value established for each area is compared with the quality color models to establish the degree of similarity of each area and the amount of localized fat in the sample. This information is stored in the results data file, classified by the processing date.

To date, about 500 samples have been processed with the CMVS, in a preliminary stage entailing fine tuning or calibration of the benchmark samples for the different quality grades. This selection is being carried out on the tuna processing line by the expert graders. 

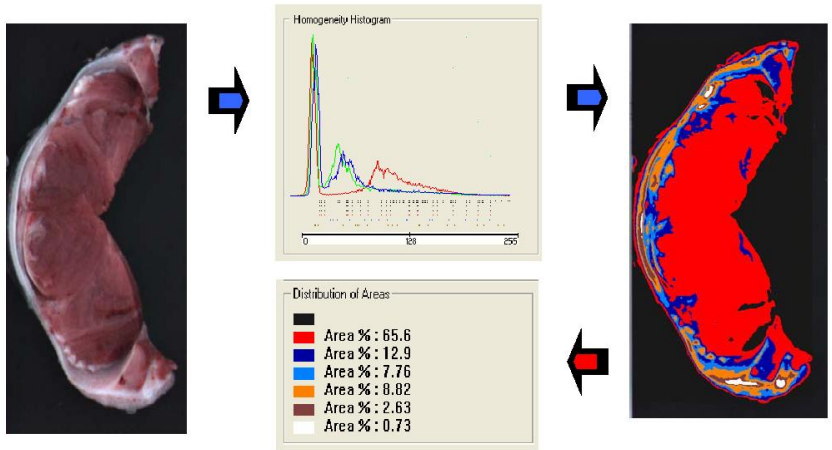

Fig. 5. Image segmentation process to determine homogeneous color areas in tail-type sample.

\section{RESUltS}

Analytical techniques based on JND and GLCM have produced quite good results; in most cases they give similar estimations of the quality model that best fits the target sample. Fig. 6 and Fig. 7 show the results of quality assessment of a random sample. Both techniques estimated that the quality of the target sample was closest to model 9. The highest value in Fig. 6 and the smallest JND value in Fig. 7 indicate which model or quality index best fits the target sample.

Table I shows the data from random analysis of a tuna sample. The table compares the sample with the quality model data set and gives the results for the $\Delta \mathrm{E}$ parameter and the GLCM single and multichannel technique [4] applied to the channels of the RGB and Lab color spaces, besides the results of the Wavelet and LBP (Local Binary Pattern) techniques used. Below, it records the uniform color distribution and the size of the tail-type sample.

By means of image interpolation prior to estimation of the GLCM, it is possible to appreciate features of the image texture more clearly and to enhance the discriminative power of GLCM modeling thanks to augmentation of the image pixel data $[15]$.

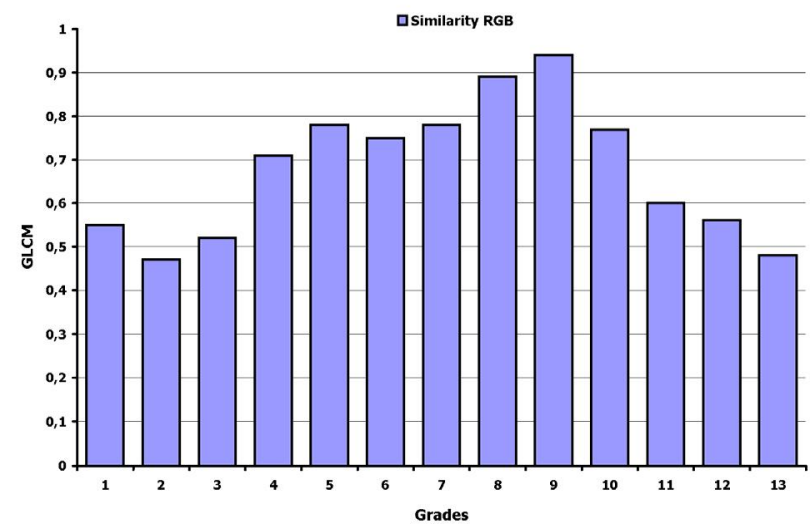

Fig. 6. Similarity of a sample to GLCM (gray level co-occurrence matrix) quality grade models.

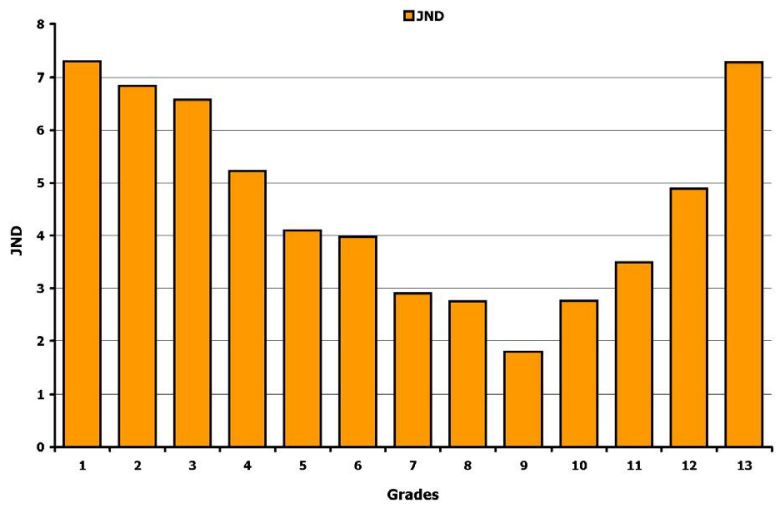

Fig. 7. Value of distance $\operatorname{JND}(\Delta \mathrm{E})$ of sample color with respect to quality grades.

The analysis performed by the CMVS is highly dependent on the production of models that accurately characterize the different quality grades in tuna captures. With the models defined to date, the reliability of classification achieved has been approximately $75 \%$.

Quality grades are assigned on a scale of 0 to 5 , the most common values being from 2.5 to 4.5 . Depending on the desired level of accuracy in classification, one sample should be modeled for every quality interval in the scale.

Table II shows a sample of the data gathered so far. These include the quality estimations of a set of tuna samples by expert graders at the Gines Mendez España S.L. (GME) tuna processing plant, the CMVS quality results and other data from the processing plant. The Quality and Fat values represent the visual inspection data provided by the expert graders.

TABLE I

ANALYTICAL DATA FOR TUNa SAMPLES EVALUation

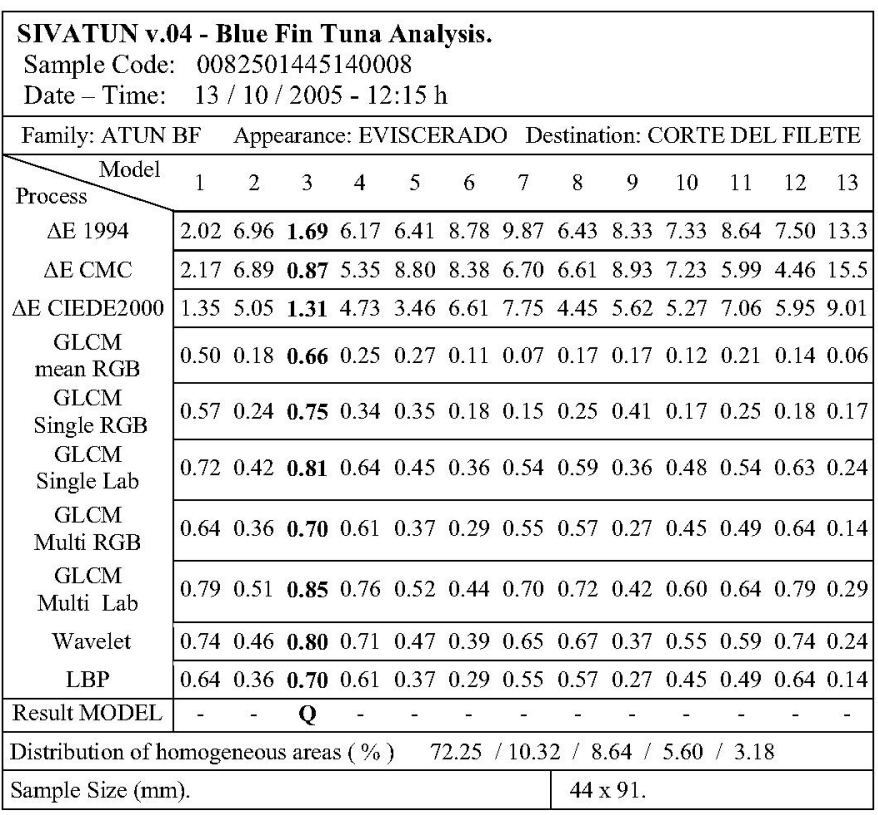


TABLE II

DATA ON TUNA CATCHES GATHERED IN THE FACTORY PROCESSING STAGE

\begin{tabular}{|c|c|c|c|c|c|c|c|c|c|}
\hline Origin \& Lot Number & Gross weight (kg) & Tag \# & Net weight $(\mathrm{kg})$ & Quality & Fat & Model & CMVS Quality & CMVS Fat $(\%)$ & CMVS Model \\
\hline SP HG LT 5.693 & 124.3 & 141.625 & 112.4 & 3.4 & 3.5 & 09 & 3.6 & $18 \%$ & 10 \\
\hline SP HG LT 5.693 & 58.2 & 141.688 & 49.8 & 3.8 & 3.6 & 11 & 3.8 & $16 \%$ & 11 \\
\hline SP HG LT 5.693 & 79.2 & 141.650 & 75.8 & 3.7 & 3.2 & 11 & 3.8 & $20 \%$ & 11 \\
\hline SP HG LT 5.694 & 128.4 & 141.628 & 114.0 & 4.0 & 3.4 & 12 & 4.2 & $16 \%$ & 13 \\
\hline SP HG LT 5.694 & 127.5 & 141.637 & 114.4 & 3.8 & 3.2 & 11 & 4.0 & $18 \%$ & 12 \\
\hline SP HG LT 5.693 & 180.3 & 141.719 & 172.4 & 4.3 & 3.4 & 13 & 4.2 & $15 \%$ & 13 \\
\hline SP HG LT 5.693 & 163.4 & 141.615 & 151.4 & 3.9 & 3.4 & 12 & 4.0 & $17 \%$ & 12 \\
\hline SP HG LT 5.694 & 81.3 & 141.640 & 72.4 & 3.5 & 3.2 & 09 & 3.4 & $19 \%$ & 09 \\
\hline SP HG LT 5.694 & 83.7 & 141.635 & 74.2 & 4.3 & 3.4 & 13 & 4.0 & $15 \%$ & 12 \\
\hline SP HG LT 5.695 & 83.7 & 141.645 & 174.2 & 3.4 & 3.2 & 10 & 3.4 & $18 \%$ & 10 \\
\hline & & $\ldots$ & $\ldots$ & .. & $\ldots$ & . & $\ldots$ & $\ldots$ & $\ldots$ \\
\hline
\end{tabular}

CMVS Quality and CMVS Fat represent the results generated by CMVS analysis. Model and CMVS values indicate the quality model to which the assessment is closest.

All this information goes into a global database at GME for traceability and processing control of its products. Examples of the analytical data thus generated are shown in Tables I and II.

Fig. 8 shows the assessment results for ten images of ten different tuna samples. It compares the quality grades assigned by the expert graders with the CMVS quality analysis. The results of this analysis are quite close to the those of the expert graders, with a maximum difference of just one point in some cases. This indicates that it is feasible to use the proposed method developed for the CMVS in determining the quality of fresh tuna meat.

\section{CONCLUSIONS}

This system proposes a method of determining the quality of fresh tuna meat by means of image analysis of tuna samples, in which the most important parameter is the predominant color of the samples.

The data provided by expert tuna meat graders have been used to model a set of color and color texture parameters exhibited by benchmark tuna samples.

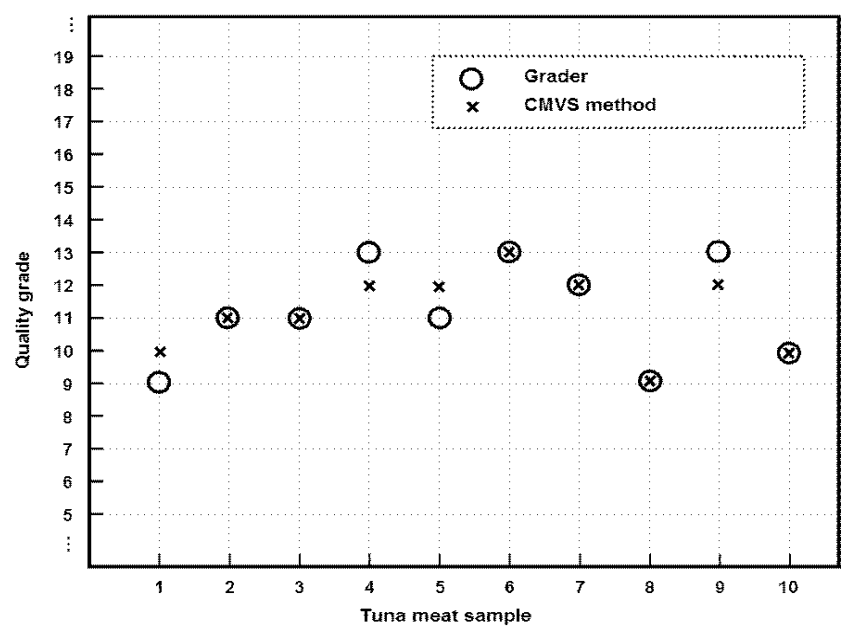

Fig. 8. Comparison of experimental results, showing the expert graders' assessments and the results generated by the CMVS analytical algorithms and techniques.
The proposed quality grading method is based on algorithmic techniques, JND( $\triangle \mathrm{E})$, GLCM and Homogeneity Histogram, combined with suitable lighting systems.

In this way a system has been developed that is capable of analyzing the color and color texture of fresh BFT samples representative of the whole tuna, so as to produce a set of data relating to quality and fat distribution.

\section{ACKNOWLEDGMENT}

This work was made possible by the financial support of the Department of Science, Technology, Industry and Trade of the Region of Murcia, through project SIVATUN-2I02SIU0020, and also by the invaluable cooperation of Gines Mendez España S.L. factory staff and the Oceanographic Spanish Institute, without whom the system could not have been developed.

\section{REFERENCES}

[1] Ling, P.P., V.N. Ruzhitsky, A.N. Kapanidis, T.C. Lee., 1996. "Measuring the color of food." Chemtech 26(3),46-52.

[2] Cheng, H., Sun,Y., 2000. "A hierarchical approach to color image segmentation using homogeneity." IEEE Transactions on Image Processing., Vol.9, No $12,2071-2082$.

[3] Luzuriaga, D.A., M.O. Balaban, S. Yeralan., 1997. Analysis of visual quality attributes of white shrimp by machine vision. Journal of Food Science., 62, 113-118, 130.

[4] Palm, C., 2004.Color texture classification by integrative co-occurrence matrices. Journal of Pattern Recognition Society. 37, 956-976.

[5] Pietikäinen, M., Ojala, T., Xu, Z. (2000). Rotation-invariant texture classification using feature distributions. Elsevier Journal of Pattern Recognition Society. 33, 43-52

[6] Wallat, G.K., Luzuriaga, D.A., Balaban, M.O., Chapman, F.A., 2002. Analysis of skin color development in live goldfish using a color machine vision system. North American Journal of Aquaculture., 64, 19-84.

[7] Balaban, M. O., S. Yeralan, Y. Bergmann., 1994. Determination of count and uniformity ratio of shrimp by machine vision. Journal of Aquatic Food Product Technology, 3, 43-58.

[8] Galaz, J. M.; Norita, T. and Albaladejo, J. (2001). Incidencia de la aparición de B.T.S. Bumt Tuna Syndrome en el cultivo y engorde de atún rojo Thunnus thynnus thynnus. Actas del VIII Congreso Nacional de Acuicultura, Santander, Dirección General de Pesca y Alimentación. Gobierno de Cantabria: 35 - 36

[9] Haralick, R.M., Shanmugan, M., Dinstein, I., 1973. Texture features for image classification. IEEE Transaction on System Man Cybernetics., $3(6), 610-621$.

[10] Hatano, M., Takahashi K., Onishi A., Kameyama Y., 1989. Quality standardization of fall chum salmon by digital image processor. Nippon Suisan Gakkaishi, 55, 1427-1433. 
[11] Jerret, A.J., Stevens, J., Holland, A.J., 1996. Tensile properties of white muscle in rested and exhausted king salmon Oncorhynchus tshawytscha. Journal of Food Science. 61, 527-532.

[12] Lowe, T.E., Ryder, J.M., Carragher, J.F., Wells, R.M.G., 1993. Flesh quality in snapper, Pagrus auratus, affected by capture stress. Journal of Food Science., 58, 770-773, 796.

[13] Mateo, A.; Villarejo, J. A.; Soto, F.; Roca-Dorda, J.; Roca Jr, J.; GarciaGomez, A.; De La Gandara, F. And Atunes De Mazarron, S. L. (2003). Electrofishing techniques: Slaughtering and quality analysis for BFT. Cah.Options Meditèrr. 60 : 113-115.

[14] Miyake, P. M.; De La Serna, J. M.; Di Natale, A.; Farrugia, A.; Katavic, I.; Miyabe, N. and Ticina, V. (2003). General review of bluefin tuna farming in the Mediterranean area. Collective Volume of Scientific Papers ICCAT. 55(1) : 114- 124.

[15] Pietikäinen, M., Ojala, T., Xu, Z. (2000). Rotation-invariant texture classification using feature distributions. Elsevier Journal of Pattern Recognition Society. 33, 43-52.

[16] Sharma, G., Trussell, H.J., 1997. Digital color imaging. IEEE Transactions on Image Processing., Vol.6, $\mathrm{N}^{\circ} 7,901-932$.

[17] Shiranita, K., Hayashi, K., Otsubo, A., Miyajima, T., Takiyama, R., 1999. Grading meat quality by texture analysis. IEEE International Conference on Systems, Man, and Cybernetics, 1999. Volume: 2, 958 -962 vol.2

[18] Skrede, G., E. Risvik, M. Huber, G. Enersen, L. Blumlein., 1990. Developing a color card for raw flesh of astaxanthin-fed salmon. Journal of Food Science 55, 361-363. 